\title{
Mechanism of Propofol's Action on MAP Rich Tubulin and Actin - An In Vitro Study
}

Sahni $\mathbf{P}^{1 *}$, Kumar $\mathbf{M}^{2}$ and Pachauri $\mathbf{R}^{3}$

${ }^{1}$ Department of Chemistry and Centre for Consciousness Studies, Dayalbagh Educational Institute, Delhi, India

${ }^{2}$ Department of Chemistry, JNU, New Delhi, India

${ }^{3}$ Department of Chemistry, Dayalbagh Educational Institute, New Delhi, India

\begin{abstract}
Despite a century of sustained research, brain scientists remain ignorant of the workings of the three pound organ that is the seat of all conscious human activity. Many have tried to attack the problem by examining the nervous systems of simpler organisms. The difficulty in establishing a link between biology and behaviour in humans is still more acute. There are techniques which are used to record the activity of single neurons in living humans. Such breakthrough methods could, in principle, begin to bridge the gap between the firing of neurons and cognition: perception, emotion, decision making and ultimately, consciousness itself. Deciphering the exact patterns of brain activity that underlie thinking and behavior will also provide critical insights into what happens when neural circuitry malfunctions in psychiatric and neurological disorders-schizophrenia, autism, Alzheimer's or Parkinson's. Anesthetics are also known to inhibit neuronal fast anterograde axoplasmic transport (FAAT) in a reversible and dose-dependent manner, but the precise mechanism by which anesthetic prevent consciousness remains unknown largely because the mechanism by which brain physiology produces consciousness is unexplained. In the present study we have used circular dichroism spectroscopy and confocal laser scanning microscopy to see the effect of propofol on the assembly of neuronal tubulin and actin together and probed into the changes of their secondary structures.
\end{abstract}

Keywords: Microtubules; Actin; Propofol interaction; Protein secondary structure

\section{Introduction}

Despite a century of sustained research, brain scientists remain ignorant of the workings of the three pound organ that is the seat of all conscious human activity. Many have tried to attack the problem by examining the nervous systems of simpler organisms. The difficulty in establishing a link between biology and behaviour in humans is still more acute. There are techniques which are used to record the activity of single neurons in living humans. Such breakthrough methods could, in principle, begin to bridge the gap between the firing of neurons and cognition: perception, emotion, decision making and ultimately, consciousness itself. Deciphering the exact patterns of brain activity that underlie thinking and behavior will also provide critical insights into what happens when neural circuitry malfunctions in psychiatric and neurological disorders- schizophrenia, autism, Alzheimer's or Parkinson's.

The current endeavor of neuroscience and indeed of philosophy, is to discover how the material brain gives rise to conscious thought. Now a day's many eminent scientist and researchers are involved in decoding the mystery of consciousness. They are mapping brain of Franciscan nuns, Buddhist monks and many others during prayer to explain spirituality or consciousness. Now the question is can a powerful brain imaging technology explain consciousness which is immaterial in nature. Many eminent researchers believe that consciousness continues even when the brain is not functioning. In other words, consciousness is something separate from the physical brain and body. Also from a scientific viewpoint, consciousness is a function of the brain. Since the brain is a material entity, consciousness is subject to the study of science. The human brain is a complex mass of tissue endowed with extraordinary capabilities. There are about 100 billion neurons in the human brain. Each neuron has synapses which are of the order of 1000 and then there are 100 operations or so per second per synapse. So the capability of the human brain is $10^{16}$ operations per second. On the other hand, a single neuron is credited by neural scientists, with capability as close to something of the order of $10^{15}$. However, neurological studies have shown that there is a sub-neuronal structure, there are as many as $10^{8}$ microtubules inside each neuron and these microtubules can operate at speed of $10^{7}$ per second. So each neuron has its capability increased by way of computational capability by a factor of $10^{15}$, given $10^{8}$ subneuronal particles known as microtubules and each microtubule has an operational speed of $10^{7}$ per second. So it brings these speeds into the realm of quantum theory. It becomes something of the order of $10^{26}$ operations per second in the human brain. So this has gone up by the factor of $10^{10}$ which is much farther than what has been achieved in Artificial Neural Networks.

Microtubules serve not only as "bone-like" support and circulatory system of living cells but also as their nervous system. The latter includes information processing, signalling and regulating its environment, for example MAP architecture and synaptic formation. Cellular automata provide a broad range of possibilities for information processing and signalling in microtubules [1]. Human consciousness is non-computable (Godel's incompleteness theorem) [2]. Human consciousness can perform "non-computable" function, furthermore no computable theory can describe it (Penrose). Non-computability to be provided by the collapse of the wave function driven by the quantumgravitational effect, the so called objective reduction [3]. According to orchestrated objective reduction (Orch-OR) theory of consciousness

*Corresponding author: Sahni P, Department of Chemistry and Centre for Consciousness Studies, Dayalbagh Educational Institute, Delhi, India, Tel: 05622801545; E-mail: deipushpasahni@gmail.com

Received: September 04, 2017; Accepted: October 04, 2017; Published October 12, 2017

Citation: Sahni P, Kumar M, Pachauri R (2017) Mechanism of Propofol's Action on MAP Rich Tubulin and Actin - An In Vitro Study. Biochem Anal Biochem 6: 338. doi: 10.4172/2161-1009.1000338

Copyright: @ 2017 Sahni P, et al. This is an open-access article distributed under the terms of the Creative Commons Attribution License, which permits unrestricted use, distribution, and reproduction in any medium, provided the original author and source are credited. 
given by Penrose and Hameroff, consciousness emerges from the brain and focus particularly on complex computation at synapses that allow communication between neurons. Neurons possess a highly organized arrangement of microtubules due to a high number of specialized nonmotor microtubule associated proteins (MAPs) with nerve cells. MAP 1A, MAP 1B, MAP2 and Tau are localized within axons and dendrites of neurons. It has been suggested that the different microtubule organizations within axons and dendrites may play a role in neuronal signalling. Evidences indicate that links exist between microtubule activity and cognitive functions by showing that during the period when the formation of synapses and visual learning occur at their highest rates the visual cortex of the brain produces massive amounts of tubulin [4]. Recently the link between microtubules and cognition has been illustrated by studies involving patients with Alzheimer's disease [5]. It has been shown that Alzheimer's compromises axonal transport indicating a link to MTs since MTs are responsible for the transport of material from the soma to the axon terminal. It has been suggested that MTs can provide a non-selective mechanism for general anesthetics. Microtubules are built of polarized proteins and are called electret polymer. The tubulin heterodimers have a hydrophobic pocket at the stitch between the two monomers. The monomers in a protofilament are 4.0-4.2 $\mathrm{nm}$ apart. Tubulin heterodimers in a microtubule can exist in two different conformational states: the alpha state and the beta state. The energy of the beta conformation state is by $0.4 \mathrm{eV}$ higher than of the alpha state. Microtubules self-organize when it undergoes a conformational change and during this process quantum vibrations are also expected to be generated in microtubules from the $\mathrm{MHz}$ to $\mathrm{GHz}$ region. Several experimental observations even suggest that cytoskeleton might be involved in cognition. For example, MAP-2, a dendritic specific, MT-cross linking MAP, has an important position in models of learning and memory in mammalian hippocampal cortex. There is also evidence that more directly links microtubules with cognitive functions. For example, microtubule activity and tubulin production correlate with peak learning, memory and experience in baby chick brains. Selective damage of microtubules in animal brains by the drug colchicine causes defects in learning and memory which mimic the symptoms of Alzheimer's disease. Alzheimer's disease itself is caused by entanglement of neuronal cytoskeleton which is suggested to be a result of microtubule dysfunction. Anesthetics are known to inhibit neuronal fast anterograde axoplasmic transport (FAAT) in a reversible and dose-dependent manner, but the precise mechanism by which anesthetic prevent consciousness remains unknown largely because the mechanism by which brain physiology produces consciousness is unexplained [6]. Propofol (an anesthetic) also changes the cytoskeletal function in cultured neuron. In fact, true consciousness is a meta-physical process i.e., the science of beyond physical material plain which should not be confused with the science of biophysical brain. Hence, it becomes important to evolve a science of consciousness by understanding the quantum approaches and integrating eastern and western perspectives of consciousness [7]. Propofol (an anesthetic) also changes the cytoskeletal function in cultured neuron. Many scientists have proposed different mechanism for the action of anesthetics [8-10].

This paper is in continuation of my already published work [11]. In the present work, efforts have been made to understand the effect of propofol (anesthetic) on complex behaviour of microtubule associated protein (MAP) rich tubulin and actin together with the help of circular dichroism spectroscopy and Confocal laser scanning microscopy. In an attempt to understand how the role of microtubule and anesthetic (propofol) action mechanism can evolve a science of consciousness, we have carried out such experiments. For this study, we have chosen propofol as an anesthetic. Chemically, propofol is 2,6- diisopropyl phenol and has been proposed to have several mechanisms of action, both through potentiation of GABAA receptor activity, thereby slowing the channel-closing time, and also acting as a sodium channel Blocker. EEG research upon those undergoing general anesthesia with propofol finds that it causes a prominent reduction in the brain's information integration capacity at gamma wave band frequencies. It has $95-99 \%$ protein binding affinity and its half-life is $30-60$ minutes [11].

\section{Materials and Methods}

MAP rich Tubulin, GTP, General Actin Buffer and General Tubulin Buffer and fluorescent reporter buffer are supplied by Cytoskeleton Inc. Denver, CO. USA.

\section{Preparation of buffer T}

GTP stock solution $(100 \mathrm{mM})$ is added to General Tubulin Buffer (80 mM PIPES pH 6.9, 2 mM MgCl, $0.5 \mathrm{mM}$ EGTA) at a final concentration of $1 \mathrm{mM}$ GTP. The buffer T will be stable for 2-4 hours on ice.

\section{Fluorescent reporter buffer}

Fluorescent reporter buffer contains $80 \mathrm{mM}$ Piperazine-N,N'-bis $\{2$ ethanesulfonic acid $\}$ seuisodium salt; $2.0 \mathrm{mM}$ Magnesium chloride; 0.5 $\mathrm{mM}$ Ethylene glycol-bis (b-amino-ethyl ether) N,N,N',N'-tetra-acetic acid, pH 6.9, $10 \mu \mathrm{M}$ DAPI (4', 6-diamidino-2-phenyindole).

\section{MAP rich tubulin reconstitution}

$1 \mu \mathrm{g}$ of lyophilized MAP rich tubulin is resuspended in $200 \mu \mathrm{l}$ General Tubulin Buffer at $0-4^{\circ} \mathrm{C}$. The reconstituted MAP rich tubulin solution is not stable and needs to be used soon after its preparation. Working solutions were prepared from the stock solution.

\section{Non-muscle actin}

$25 \mu \mathrm{l}$ distilled water was added to $250 \mu \mathrm{g}$ of lyophilised nonmuscle actin protein. Propofol (2,6-di-isopropylphenol) (Anesthetic) is supplied by MP Biomedicals, Mumbai. $100 \mathrm{mM}$ Propofol was used in the study.

\section{General actin buffer}

Procured from Cytoskleton Inc., USA. Throughout the experiments, all solutions were prepared in MilliQ water. $\mathrm{pH}$ measurements were carried out using EUTECH instruments-pH 510.

\section{Circular dichroism (CD) measurements}

The isothermal studies of Tubulin by CD measurements were carried out with Chirascan, a polarimeter of Applied Photophysics equipped with a QUANTUM N.O.R.T.H.W.E.S.T.-TC125, a Peltiertype temperature controller. The instrument was calibrated with d-10camphorsulfonic acid. All the isothermal CD measurements were made at $25^{\circ} \mathrm{C}$. Spectra were collected with $20 \mathrm{~nm} / \mathrm{min}$ scan speed, $0.1 \mathrm{~nm}$ data pitch, and a response time of $2 \mathrm{~s}$. Each spectrum was the average of 10 scans. The Far-UV CD spectra $(200-260 \mathrm{~nm})$ were taken at protein concentrations of $0.889 \mathrm{mg} / \mathrm{ml}$ in a cell of $0.1 \mathrm{~cm}$ path length. All spectra were smoothed by the Savitzky-Golay method with 25 convolution width. CD values $(\theta)$ in mdeg, were obtained from the instrument readings. Polymerization of tubulin protein is carried out using standard protocols with slight modifications [11-17]. Circular dichroism study was carried out after the polymerization has taken place. 


\section{Confocal laser scanning microscopy (CLSM)}

The polymerisation studies and effect of anaesthetic (propofol) on MAP-rich Tubulin, Non-Muscle Actin separately and MAP-rich Tubulin along with actin (complex system) were carried out with FLUOVIEW FV 1000 CONFOCAL LASER SCANNING BIOLOGICAL MICROSCOPE. The samples were made into permanent glass slides by pouring the sample on the glass slide and mounting with glycerine and covering with a cover slide. For this experiment, a LASER having its central wavelength at $375 \mathrm{~nm}$ was used as the source for exciting the DAPI (4', 6-diamidino-2-phenyindole) present in fluorescent buffer. Emission was subsequently collected at $440 \mathrm{~nm}$ through a single monochromator with a $5 \mathrm{~nm}$ band pass over a total time range (TAC) of $100 \mathrm{~ns}$ for all samples. Emission polarizer was set at 55.4 degree magic angle to exclude rotational anisotropy lifetime decay to simple decay life time data. Emission decays were fit with appropriate instrument response functions (IRF) collected using a scattering solution. The FWHM (full width at half-maximum) of the IRFs collected was typically in the range of $\sim 120$ ps.

In order to understand the chemistry of MAP-rich Tubulin binding and changes in its secondary structure \& tertiary structure, we carried out our studies using standard protocol and methods reported in the literature as general with slight modifications [11,12,14-16]. Here we have not considered conditions ideal for quantum brain structure relevant to consciousness but our future endeavour would be to do experiments under conditions ideal for quantum brain structure relevant to consciousness.

In human brain neurons, actin proteins are also present along with intermediate filaments and microtubules, so in the present study we have also probed into the secondary structure of actin protein and action of propofol on actin and MAP rich tubulin polymerisation. Effect of propofol on individual proteins (simple system) and when taken together (complex system) also reflected the mechanism of anesthetic action.

\section{Results and Discussion}

The reported total composition of the secondary structure in $\alpha$,tubulin is $\alpha$-helices $(40.5 \%), \beta$-sheets $(13 \%)$ and random coil $(46.5 \%)$ and other secondary structures (parallel $\beta$-strands, antiparallel $\beta$-sheets). In the conformation of tubulin, the $\alpha$-helices are responsible for nucleation and the rate of polymerization while antiparallel $\beta$-sheets serve to regulate these functions as well as attribute to the stability of such a dynamic protein [17-23].

Secondary structures of MAP rich tubulin and actin proteins were studied using Circular Dichorism measurements. We took tubulin glycerol buffer and general actin buffer as control for various reaction sets.

Sample 1 data show the CD data for the sample containing MAPrich tubulin without propofol. The $\alpha$ - helix and $\beta$ - turn in this reaction condition (MAP rich tubulin in presence Guanosine Tri Phosphate (GTP) and Tubulin Glycerol Buffer) were found to be $13.30 \%$ and $57.50 \%$ respectively. On adding propofol to this reaction conditions (Sample 2), the increase in the values of $\alpha-$ helix and $\beta$ - turn were found to be from 13.30 to $13.40 \%$ and $57.50-57.60 \%$ respectively. And there is also reduction in antiparallel content from 135.80 to $135.60 \%$.

From these data, it is interfered that propofol binds with the hydrophobic pockets of the tubulin heterodimer via weak van der Waals' forces and changes the secondary structure of MAP rich tubulin protein [24-26].

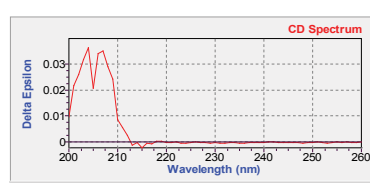

\begin{tabular}{|l|r|r|r|r|}
\hline $\begin{array}{l}\text { Sample } \\
\text { I }\end{array}$ & $\begin{array}{l}\mathbf{2 0 0 - 2 6 0} \\
\mathbf{n m}\end{array}$ & $\begin{array}{l}\mathbf{2 0 5 - 2 6 0} \\
\mathbf{n m}\end{array}$ & \multicolumn{1}{|l|}{$\begin{array}{l}\mathbf{2 1 0 - 2 6 0} \\
\mathbf{n m}\end{array}$} & \multicolumn{1}{|c|}{ Total } \\
\hline Helix & $6.30 \%$ & $3.40 \%$ & $3.60 \%$ & $\begin{array}{r}13.30 \\
\%\end{array}$ \\
\hline $\begin{array}{l}\text { Antipara } \\
\text { llel }\end{array}$ & $43.40 \%$ & $45.50 \%$ & $46.90 \%$ & $\begin{array}{r}135.80 \\
\%\end{array}$ \\
\hline Parallel & $5.60 \%$ & $5.20 \%$ & $5.30 \%$ & $\begin{array}{r}16.10 \\
\%\end{array}$ \\
\hline $\begin{array}{l}\text { Beta- } \\
\text { Turn }\end{array}$ & $19.30 \%$ & $18.90 \%$ & $19.30 \%$ & $\begin{array}{r}57.50 \\
\%\end{array}$ \\
\hline $\begin{array}{l}\text { Random. } \\
\text { Coil }\end{array}$ & $36.40 \%$ & $34.90 \%$ & $35.30 \%$ & $\begin{array}{r}106.60 \\
\%\end{array}$ \\
\hline $\begin{array}{l}\text { Total } \\
\text { Sum }\end{array}$ & $111.00 \%$ & $107.90 \%$ & $110.50 \%$ & $\begin{array}{r}329.40 \\
\%\end{array}$ \\
\hline
\end{tabular}

Sample 1: CD spectra and data of secondary structures content for MAP rich Tubulin in $1 \mathrm{mM}$ GTP without propofol.

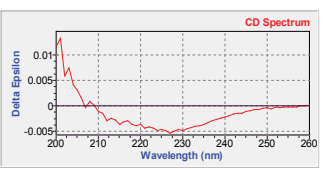

\begin{tabular}{|l|r|r|r|r|}
\hline Sample II & \multicolumn{1}{l|}{$\begin{array}{l}\mathbf{2 0 0 - 2 6 0} \\
\mathbf{n m}\end{array}$} & \multicolumn{2}{l|}{$\begin{array}{l}\mathbf{2 0 5 - 2 6 0} \\
\mathbf{n m}\end{array}$} & \multicolumn{2}{l|}{$\begin{array}{l}\mathbf{2 1 0 - 2 6 0} \\
\mathbf{n m}\end{array}$} & \multicolumn{1}{l|}{ Total } \\
\hline Helix & $6.30 \%$ & $3.40 \%$ & $3.70 \%$ & $13.40 \%$ \\
\hline $\begin{array}{l}\text { Antiparalle } \\
1\end{array}$ & $43.30 \%$ & $45.40 \%$ & $46.90 \%$ & $\begin{array}{r}135.60 \\
\%\end{array}$ \\
\hline Parallel & $5.60 \%$ & $5.20 \%$ & $5.30 \%$ & $16.10 \%$ \\
\hline Beta-Turn & $19.30 \%$ & $19.00 \%$ & $19.30 \%$ & $57.60 \%$ \\
\hline $\begin{array}{l}\text { Random. } \\
\text { Coil }\end{array}$ & $36.40 \%$ & $34.90 \%$ & $35.30 \%$ & $\begin{array}{r}106.60 \\
\%\end{array}$ \\
\hline Total Sum & $110.90 \%$ & $107.90 \%$ & $110.40 \%$ & $\begin{array}{r}329.20 \\
\%\end{array}$ \\
\hline
\end{tabular}

Sample 2: CD spectra and data of secondary structures content for MAP rich tubulin in $1 \mathrm{mM}$ GTP with $100 \mathrm{mM}$ propofol.
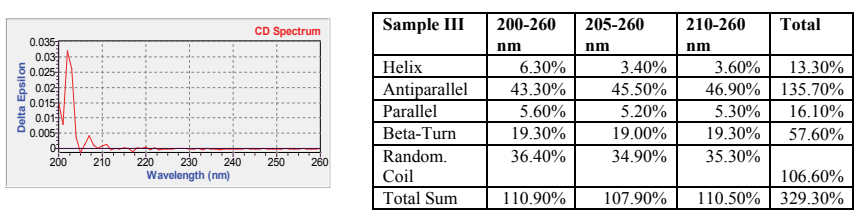

Sample 3: CD spectra and data of secondary structures content for non-muscle actin in general actin buffer without propofol.

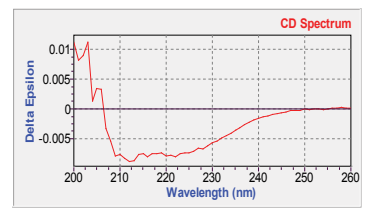

\begin{tabular}{|c|c|c|c|c|}
\hline $\begin{array}{l}\text { Sample } \\
\text { IV }\end{array}$ & \begin{tabular}{|l|}
$200-260$ \\
$\mathrm{~nm}$
\end{tabular} & $\begin{array}{l}\text { 205-260 } \\
\mathrm{nm}\end{array}$ & \begin{tabular}{|l|}
$210-260$ \\
$\mathrm{~nm}$
\end{tabular} & Total \\
\hline Helix & $6.30 \%$ & $3.40 \%$ & $3.70 \%$ & 13.40 \\
\hline $\begin{array}{l}\text { Antiparal } \\
\text { lel }\end{array}$ & $43.20 \%$ & $45.40 \%$ & $46.80 \%$ & $\begin{array}{r}\% \\
135.40 \\
\%\end{array}$ \\
\hline Parallel & $5.60 \%$ & $5.20 \%$ & $5.30 \%$ & 16.10 \\
\hline $\begin{array}{l}\text { Beta- } \\
\text { Turn }\end{array}$ & $19.30 \%$ & $19.00 \%$ & $19.30 \%$ & $\begin{array}{r}57.60 \\
\%\end{array}$ \\
\hline $\begin{array}{l}\text { Random. } \\
\text { Coil }\end{array}$ & $36.40 \%$ & $34.90 \%$ & $35.30 \%$ & $\begin{array}{r}106.60 \\
\%\end{array}$ \\
\hline $\begin{array}{l}\text { Total } \\
\text { Sum }\end{array}$ & $110.90 \%$ & $107.80 \%$ & $110.40 \%$ & $\begin{array}{l}329.10 \\
\%\end{array}$ \\
\hline
\end{tabular}

Sample 4: $C D$ spectra and data of secondary structures content for nonmuscle actin in general actin buffer with $100 \mathrm{mM}$ propofol.

Since human brain is a complex system and each neurons in human brain contains microtubule, actin, intermediate filaments along with other biomolecules. Attempt has been made to understand the effect of anesthetic on individual actin protein (simple system) and a little more complex system (i.e., actin and tubulin together).

Sample 3 data show the CD data of the Non-muscle Actin without Propofol. The changes in secondary structure of actin protein were observed when propfol was added to actin protein. The helix and $\beta$ - turn contents in actin protein without propofol were found to be $13.30 \%$ and $57.60 \%$ respectively. The antiparallel content in this reaction condition was found to be $135.70 \%$. On adding propofol to this reaction condition (Sample 4), the increase in the helix content was found to be from 13.30 to $13.40 \%$ but there is no change in beta -turn content. There is reduction in antiparallel content on adding propofol from $135.70-135.40 \%$.

From these data it is inferred that the polymerisation of actin is also affected in the presence of propofol. 
Citation: Sahni P, Kumar M, Pachauri R (2017) Mechanism of Propofol's Action on MAP Rich Tubulin and Actin - An In Vitro Study. Biochem Anal Biochem 6: 338. doi: 10.4172/2161-1009.1000338

Page 4 of 5

Sample 6 data show the CD data for MAP rich Tubulin and Non Muscle Actin without Propofol. The helix, $\beta$ - turn and antiparallel contents were found to be $13.30 \%, 57.50 \%$ and $135.80 \%$ respectively.

On adding propofol to the above reaction conditions (Sample 6), the increase in the helix content was found to be $13.40 \%$ but there is no change in beta-turn content. The antiparallel content on adding propofol has decreased from $135.80-135.50 \%$.

From these data it is inferred that the polymerisation behaviour in a complex system is also affected in the presence of propofol. There is slight increase in the helix content when propofol binds to tubulin hydrophobic pockets in the presence of actin protein [27-29].

Circular dichroism data interpretation was carried out by using CDNN 2.1 program to get the quantitative data of secondary structures

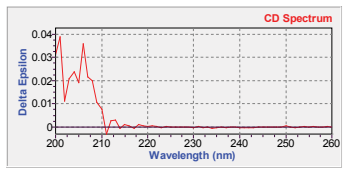

\begin{tabular}{|l|r|r|r|r|}
\hline Sample V & \multicolumn{1}{|l|}{$\begin{array}{l}\mathbf{2 0 0 - 2 6 0} \\
\mathbf{n m}\end{array}$} & $\begin{array}{l}\text { 205-260 } \\
\mathbf{n m}\end{array}$ & $\begin{array}{l}\mathbf{2 1 0 - 2 6 0} \\
\mathbf{n m}\end{array}$ & \multicolumn{1}{l|}{ Total } \\
\hline Helix & $6.30 \%$ & $3.40 \%$ & $3.60 \%$ & $13.30 \%$ \\
\hline Antiparallel & $43.40 \%$ & $45.50 \%$ & $46.90 \%$ & $135.80 \%$ \\
\hline Parallel & $5.60 \%$ & $5.20 \%$ & $5.30 \%$ & $16.10 \%$ \\
\hline Beta-Turn & $19.30 \%$ & $18.90 \%$ & $19.30 \%$ & $57.50 \%$ \\
\hline $\begin{array}{l}\text { Random. } \\
\text { Coil }\end{array}$ & $36.40 \%$ & $34.90 \%$ & $35.30 \%$ & $106.60 \%$ \\
\hline Total Sum & $111.00 \%$ & $107.90 \%$ & $110.50 \%$ & $329.40 \%$ \\
\hline
\end{tabular}

Sample 5: CD spectra and data of secondary structures content for MAP-rich tubulin and non-muscle actin in general tubulin and actin buffer without propofol.

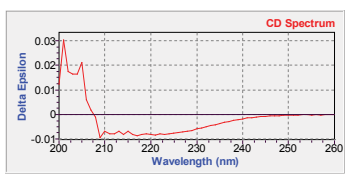

\begin{tabular}{|l|r|r|r|r|}
\hline Sample VI & \multicolumn{1}{l|}{$\begin{array}{l}\mathbf{2 0 0 - 2 6 0} \\
\mathbf{n m}\end{array}$} & $\begin{array}{l}\mathbf{2 0 5 - 2 6 0} \\
\mathbf{n m}\end{array}$ & $\begin{array}{l}\mathbf{2 1 0 - 2 6 0} \\
\mathbf{n m}\end{array}$ & \multicolumn{1}{l|}{ Total } \\
\hline Helix & $6.30 \%$ & $3.40 \%$ & $3.70 \%$ & $13.40 \%$ \\
\hline Antiparallel & $43.30 \%$ & $45.40 \%$ & $46.80 \%$ & $135.50 \%$ \\
\hline Parallel & $5.60 \%$ & $5.20 \%$ & $5.30 \%$ & $16.10 \%$ \\
\hline Beta-Turn & $19.30 \%$ & $18.90 \%$ & $19.30 \%$ & $57.50 \%$ \\
\hline $\begin{array}{l}\text { Random. } \\
\text { Coil }\end{array}$ & $36.40 \%$ & $34.90 \%$ & $35.30 \%$ & \\
\hline Total Sum & $110.90 \%$ & $107.80 \%$ & $110.40 \%$ & $329.60 \%$ \\
\hline
\end{tabular}

Sample 6: CD spectra and data of secondary structures content for MAP-rich tubulin and non-muscle actin in general tubulin and actin buffer with $100 \mathrm{mM}$ propofol.

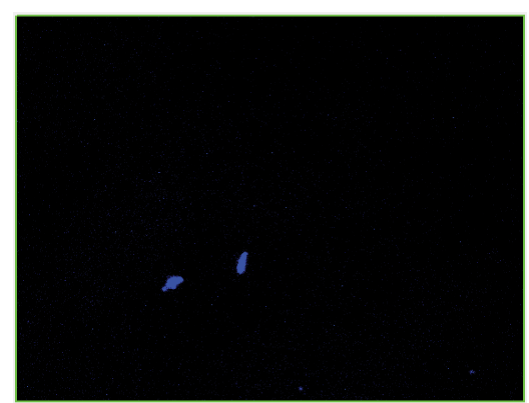

Figure 1: Confocal microscope image for MAP rich tubulin with $1 \mathrm{mM}$ guanosine tri-phosphate in tubulin glycerol buffer at 40X (Sample I).

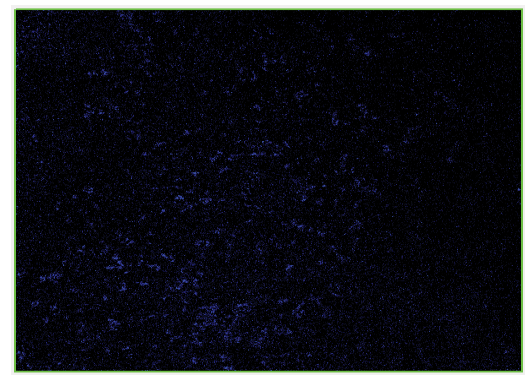

Figure 2: Confocal microscope image of MAP rich tubulin with $1 \mathrm{mM}$ GTP and $100 \mathrm{mM}$ propofol in tubulin glycerol buffer at 40X (Sample II).

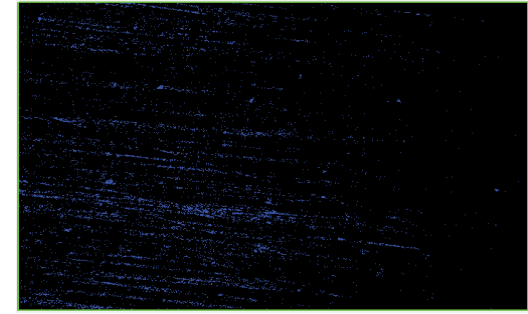

Figure 3: Confocal microscope image for MAP-rich tubulin and non-muscle actin with $100 \mathrm{mM}$ propofol and $1 \mathrm{mM}$ GTP in tubulin glycerol buffer and general actin buffer at $40 \mathrm{X}$ (Sample III).

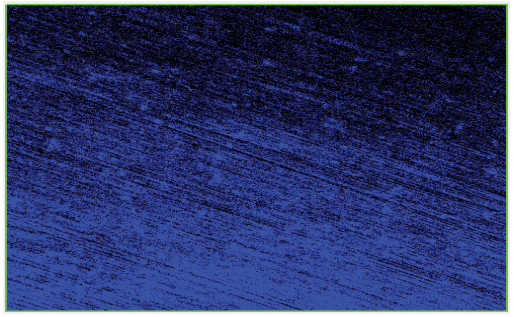

Figure 4: Confocal microscope image for MAP-rich tubulin and non-muscle actin with $1 \mathrm{mM}$ GTP in tubulin glycerol buffer and general actin buffer at $40 \mathrm{X}$ (Sample IV).

content. In order to understand the effect of anesthetic on proteins, Sample 1 (without propofol) is compared with Sample 2 (with propofol), Sample 3 (without propofol) with Sample 4 (with propofol) and Sample 5 (without propofol) with Sample 6 (with propofol).

\section{Confocal laser scanning microscopy (CLSM)}

Confocal Laser Scanning Microscopy (CLSM) for the same reaction sets were carried out to validate our results of circular dichroism measurements.

Figure 1 shows the confocal microscopic Figure of the sample containing MAP Rich Tubulin in presence of Guanosine Tri-Phosphate and Tubulin Glycerol Buffer. It shows assembly of MAP rich tubulin into microtubules but upon adding propofol to the same reaction condition, the disassembly of the microtubules into tubulin occurs (Figure 2).

This finding further confirms that the propofol binds to the tubulin hydrophobic pockets and it inhibits the assembly of tubulin into microtubules.

Almost similar trends were observed in Figures 3 and 4. CLSM figure showed a very complex polymerization behaviour when actin and MAP rich tubulin proteins were taken together (complex system) (Figure 4). On adding propofol to this complex system, the polymerization behaviour is significantly changed.

From the above observations, it is inferred that propofol affects the tubulin and actin polymerisation via weak vander waals' forces and it blocks the endogenous london dispersive forces which are necessary for clinical responsiveness. Propofol also affects secondary structures content like helix, strands, sheets, radom coil etc. when it binds with proteins (tubulin and actin). The present study also reflects that when propofol binds to the hydrophobic pocket of the protein, the secondary structures content of the protein changes which remarkably supports Hameroff's hypothesis for anesthetic mechanism. But the exact mechanism for the anesthetic action has not been reported so far which could lead us to the actual cause of cognitive behaviour/consciousness. 
Citation: Sahni P, Kumar M, Pachauri R (2017) Mechanism of Propofol's Action on MAP Rich Tubulin and Actin - An In Vitro Study. Biochem Anal Biochem 6: 338. doi: 10.4172/2161-1009.1000338

Page 5 of 5

\section{Conclusion}

Circular dichoirism measurements show that propofol affects the secondary structures of tubulin and actin which was further confirmed by confocal laser scanning microscope (CLSM). Studies show that there is not a single mechanism for anesthetic action. Propofol hinders the propagation of conformational changes along protofilaments and perhaps along helical pathways within the microtubule lattice. Microtubule population in presence of propofol is not capable of carrying out collective action. Propofol also affects actin polymerization. There is not a single mechanism by which anaesthetic acts. Anaesthetics seem to cause physical unconsciousness or clinical unresponsiveness when they block the brain's ability to integrate information.

Our future endeavour would be to determine conformational states available in tubulin molecule experimentally and what happens when neural circuitry malfunctions in patients suffering from neurodegenerative disorders.

\section{Acknowledgement}

The authors are extremely grateful to Revered Prof. P. S. Satsangi, Chairman Advisory Committee on Education, Dayalbagh Educational Institutions for incessan guidance and encouragement. I am also grateful to Advance Instrumentation Research Facility (AIRF), Jawaharlal Nehru University (JNU), New Delhi, for providing us research facility.

\section{References}

1. Hameroff (2015) A model of quantum-vo $n$ Neuman hybrid cellular automata: principles and simulation of quantum coherent superposition and decoherence in cytoskeletal microtubules. Quantum Information and Computation 15: 22-36.

2. Murawski R (1997) Godel's incompleteness theorem and computer science. Foundations of Science 2: 123-135.

3. Hameroff S, Penrose R (2007) Orchestrated reduction coherence in brain microtubules. The "Orch OR" model for consciousness. Cognitive Science 31: 1035-1045.

4. Tuszynski, Craddock (2007) On the role of the microtubules in cognitive brain functions. NeuroQuantology 5: 32-57.

5. Bjornstorm K, Eintrei C (2003) The difference between sleep and anaesthesia is in the intracellular signal. Acta Anaesthesiologica \& Scandinavica 47: 157-164.

6. Hameroff S, Watt RC, Borel JD, Carlson G (1982) General anesthetics directly inhibit electron mobility: dipole dispersion theory of anesthetic action. Physiological Chemistry and Physics 14: 183-187.

7. Satsangi PS, Sahni V, Srivastava DP (2016) Modelling microtubules in the brain as n-qudit quantum Hopfield network and beyond. Int J Gen Syst 45: 1.

8. Hinkley RE, Samson FE (1972) Anesthetic-induced transformation of axonal microtubules. J Cell Biol 53: 258-263.

9. Hinkley RE, Telser AG (1974) The effects of Halothane on cultured mouse neuroblastoma cells. J Cell Biol 63: 531-540.
10. Vergara GA, Livingston (1981) Halothane modifies colchicine binding to tubulin Pharmacology 23: 264-270.

11. Pushpa S (2016) Tubulin conformation and anaesthetic interaction -An experimental study. Biochem and Anal Biochem 5: 1

12. Bokros CL, Jeffrey DH, Hanesworth VR, Jaitra VM, Louis CM (1993) Characterization of the reversible taxol-induced polymerization of plant tubulin into microtubules. Biochemistry 32: 3437.

13. Desai MF, Pantaloni D (1997) Hydrolysis of GTP associated with the formation of tubulin oligomers is involved in the Microtubule Nucleation. Biophys $\mathrm{J} 73$ : 418-427.

14. Carlier MF, Pantaloni D (1978) Kinetic analysis of cooperativity in tubulin polymerization in the process of guanosine di- or tri-phosphate nucleotides. Biochemistry 17: 1908-1915.

15. Desai A, Mitchison TJ (1997) Microtubule polymerization dynamics. Annu Rev Cell Dev Biol 13: 83-117.

16. Gaskin F, Cantor CR, Shelenski M (1974) Turbidimetric studies of the in vitro assembly and disassembly of porcine neurotubules. J Mol Biol 89: 737-758.

17. Ventilla M, Cantor CR, Shelanski M (1972) A circular dichroism study of microtubule protein. Biochemistry 11: 1554-1561.

18. Nogales, Downing KH, Lowe J (2007) Refined structure of a-tubulin at $3.5 \AA$ resolution. J Mol Biol 113: 1045-1057.

19. Reed J, Hull WE, Ponstingl H, Himes RH (1992) Conformational properties of the beta (400-436) and beta (400-445) C-terminal peptides of porcine brain tubulin. Biochemistry 31: 11888-11895.

20. Yang Z, Lijun Z, Zu Y, Gang Y, Wu X (2009) Conformational dynamics and thermal stabilities of the $\alpha \beta$-tubulin dimer: A molecular dynamics simulation study. In Silico Biology 9: 23.

21. Greenfield NJ (2006) Using circular dichroism spectra to estimate protein secondary structure. Nat Protoc 1: 2876-2890.

22. Johnson WC (1990) Protein secondary structure and circular dichroism: A practical guide. Proteins 7: 205-214.

23. Andrzej K, Kamel K (2011) Computational study of binding of epothilone $A$ to B-tubulin. Acta Biochim Pol 58: 255-260.

24. Tabony J (2007) Self-organization and higher level emergent phenomenon in a population of microtubules. Neuroquatology 5: 100-144.

25. Satsangi PS (2011) Cosmology of consciousness: Towards quantum-theoretic systems modelling; Spirit-Mind-Brain Interactions. Vision Talk at the Inaugural Workshop of the Centre for Consciousness Studies.

26. Sahni P (2013) Chemistry of microtubules and consciousness. Lamber Academic Publishing (LAP).

27. Hameroff S (2006) The entwined mysteries of anesthesia and consciousness Anesthesiology 105: 400-412.

28. Hameroff S (1998) Anesthesia, consciousness and hydrophobic pockets Toxicology Letters 100/101: 31-39.

29. Andreu JM, Torre J, Jose LC (1986) Interaction of tubulin with octyl glucoside and deoxycholate. 2. Protein conformation, Binding of colchicine ligands and microtubule assembly. Biochemistry 25: 5230-5239. 